\title{
THE VERY LARGE HADRON COLLIDER: THE FARTHEST ENERGY FRONTIER
}

\author{
William A. Barletta, E. O. Lawrence Berkeley National Laboratory \\ University of California, Berkeley, CA 94720
}

\begin{abstract}
The Very Large Hadron Collider (or Eloisatron) represents what may well be the final step on the energy frontier of accelerator-based high energy physics. While an extremely high luminosity proton collider at $100-200$ $\mathrm{TeV}$ center of mass energy can probably be built in one step with LHC technology, that machine would cost more than what is presently politically acceptable. This talk summarizes the strategies of collider design including staged deployment, comparison with electron-positron colliders, opportunities for major innovation, and the technical challenges of reducing costs to manageable proportions. It also presents the priorities for relevant $R \& D$ for the next few years.
\end{abstract}

\section{OVERVIEW}

In the broadest sense, the VLHC (or Eloisatron) is the ultimate femtoscience experiment in that it explores the phenomena that were operative during the first 100 femtoseconds of the universe. Viewed on a scale of the energy of interaction of the quarks and gluons, the central province of accelerator-based high energy physics is represented by the shaded region between the two vertical lines in Figure 1. At the left-hand line are machines such as the Bevatron; the right hand line is the VLHC. To "look back" further into the past, i.e, at higher interaction energies, one must rely on using the universe as a laboratory via experiments to look for relic radiations such as the cosmic microwave background.

The VLHC research effort in the US is the product of a loose collaboration formed in 1998 by Fermilab, LBNL and Brookhaven with additional Steering Committee members from Cornell and SLAC. While the technical focus of the collaboration is a post-LHC hadron collider sited at Fermilab, the goal has been to craft a compelling program for 50 years of forefront high energy physics. In terms of the collider characteristics, VLHC should be a large advance beyond LHC. While the eventual goal is to reach the highest practical energy, multi-step scenarios for reaching this energy are the most realistic in fiscal terms. Eventually more than $100 \mathrm{TeV}$ per beam may be achieved using multiple rings occupying the same tunnel.

At the time of this writing VLHC is the only sure way to access the energy scale $>1 \mathrm{TeV}$. Furthermore, for a VLHC at $100 \mathrm{TeV}$ per beam, we have identified no extraordinary technical difficulties that preclude operation at $10^{35} \mathrm{~cm}^{-2} \mathrm{~s}^{-1}$ using present technologies. (To be sure, radiation damage to detectors and separation of events in collisions with more than 10 events/crossing/cm is a serious issue needing extensive research.) In fact, proton synchrotrons could reach up to $1 \mathrm{PeV}$ proton c.m. energy if a way to operate with a warm bore vacuum system can be developed. The discovery potential of VLHC far surpasses that of any conceivable lepton collider, because of the unique combination of much higher beam energy plus high luminosity. The VLHC is the only sure way to the next energy scale and we could begin its formal design now.

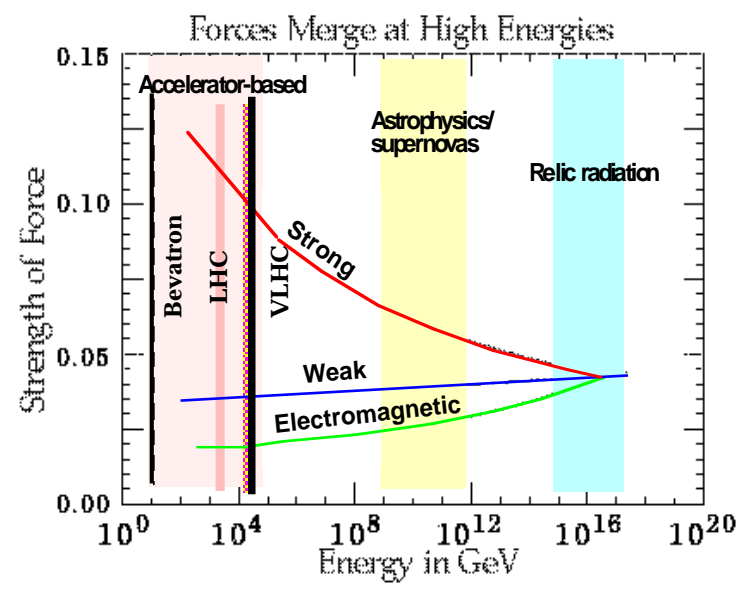

Figure 1. The Age of Accelerators in the investigation of fundamental forces.

\section{TECHNICAL ISSUES}

From the outset of the collaboration, we have considered three diverse design strategies for VLHC. The first is a low field approach (LF) using superferric magnets excited by a superconducting transmission line. This approach requires an immense tunnel to reach $100 \mathrm{TeV}$ per beam and results in extremely large stored beam energy. Even at the highest energies synchrotron radiation has minimal influence on machine design. A second approach, which has not received much detailed study, would use ductile superconductor in a RHIC-like magnet design at $4-6 \mathrm{~T}$. In this case there is some limited luminosity enhancement from radiation damping. The third option (HF) employs high field magnets with brittle superconductor such as $\mathrm{Nb}_{3} \mathrm{Sn}$ or $\mathrm{Nb}_{3} \mathrm{Al}$ operating at $>10 \mathrm{~T}$. This approach minimizes both the size of the tunnel, and the stored beam energy, but maximizes the effects of synchrotron radiation. 
Accelerator physics issues relevant to all three approaches were reviewed at SLAC in March 2001 at the VLHC Instability Workshop. The most serious potential problem appears to be the transverse mode coupling instability. The TMCI safety factor, $\mathrm{N}_{\text {thresholdr }} / \mathrm{N}_{\text {beam }}$ is 0.5 for the low field case and 8 for the high field case. This estimate is likely to be pessimistic and the instability can likely be controlled by feedback systems. With respect to the resistive wall multi- bunch instability the growth increments are $\mathrm{LF} \sim 1$ turn and $\mathrm{HF} \sim 5$ turns. This instability also can be controlled by a feedback system plus an audio frequency feed-forward system as proposed by Marriner. Among issues not expected to be serious: the electron cloud instability with growth times LF $0.25 \mathrm{~s}$ and $\mathrm{HF}-0.5-10 \mathrm{~s}$; the longitudinal microwave instability (safety factor 20); the coherent synchrotron tune-shift (safety factor $\sim 10$ ). Effects of ground motion can be suppressed by feedback. For more information see

www.slac.stanford.edu/ achao/ VLHCWorkshop.html

The dominant beam physics effect in the VLHC is synchrotron radiation. First, radiation alters the beam distribution and the allowed tune shift consistent with acceptable backgrounds. Even at $100 \mathrm{TeV} /$ beam in the high field design, the damping decrement is $\left\langle 10^{-6}\right.$. The maximum total tune shift is limited to $<0.02$. A positive effect of radiation is damping of the beam emittance; this effect increases luminosity. Perhaps it eases injection and loosen magnet tolerances, in which case it would save money. More certainly energy losses form synchrotron radiation limit the beam current due to three effects: 1 ) Direct heating of walls which leads to cryogenic heat load which may increase wall resistivity, 2) Indirect heating of the walls via two stream effects (electron cloud) which may triple or quadrupole the heat load, 3) Photodesorption of gas which may lead to beam-gas scattering which could lead to a magnet quench. Controlling these effects increases costs. Note that the direct thermal effects of synchrotron radiation scale with the radiation power (as the fourth power of $E_{\text {beam }}$ ) while the two stream effects scale as the photon number (linearly with $\mathrm{E}_{\text {beam }}$ ).

Thermal loads and photo-desorption of gas in the beam tube directly drive the design of the vacuum and cryogenic systems in the collider. The beam screen provides a critical function of intercepting the thermal load at a temperature well above the magnet temperature; the screen however increases required magnet aperture. Slots in the screen pump photodesorbed gas from the path of the beam for absorption behind the screen. This gas may be removed by 1) physical absorption by a zeolyte, which will require regeneration at $20 \mathrm{~K}$ tri-monthly, or 2) chemical absorption in a getter material, which has a finite life and which will require regeneration at $450 \mathrm{~K}$ at least annually.

An alternative that requires further study is to let the photons escape from the beam tube to strike small "fingers" at $70-100 \mathrm{~K}$ temperature protruding into the beam tube or to escape into an ante-chamber. The LHC tunnel cryogenic system has more than 1 valve per magnet average. Such superfluid systems are impractical at the scale of VLHC. Generally one concludes that scaling the LHC approach is not an option.

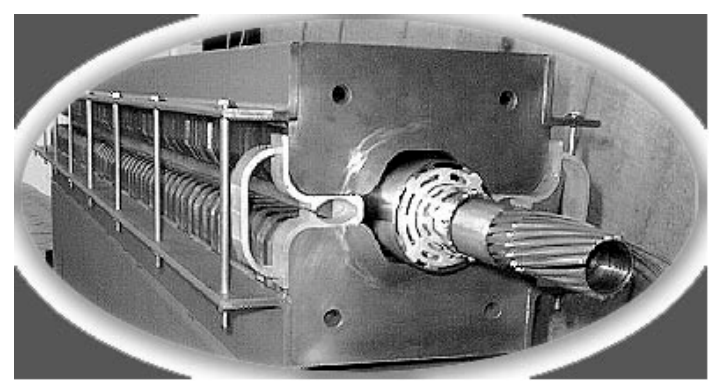

Fig.2 A model of a 2-in-1 transmission line magnet

The 2-in-1 transmission line magnet (see fig. 2) used in the low field approach lets photons in the outboard beam tube escape into a warm vacuum system. In this magnet a transmission line carrying $75-100 \mathrm{kA}$ excites the magnet. The cold mass is restricted to the transmission line which is a very low heat leak structure. The iron and the extruded $\mathrm{Al}$ beam tube plus ante-chamber are at room temperature. The B-field at the superconductor is only 1 Tesla; thus the critical current density is much higher than in the other magnet configurations.

SSC experience shows us the cost drivers for the medium and high field versions of VLHC. In SSC the main collider accounted nearly $60 \%$ of the total cost. Of this amount more than $80 \%$ was devoted to the collider dipoles. Therefore lowering dipole cost is the key to cost control.

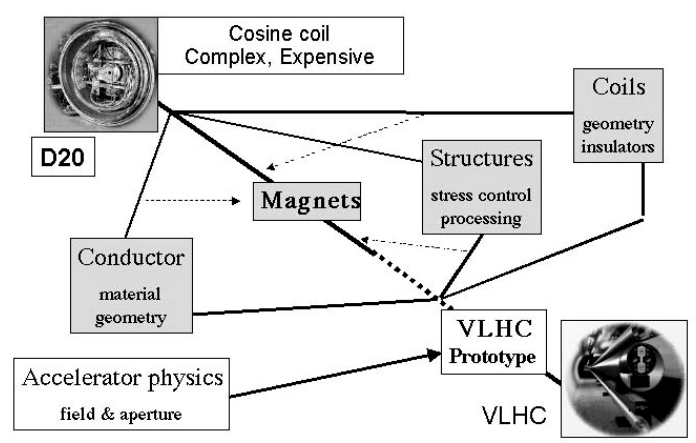

Fig. 3 Program of magnet development to control dipole costs

The US program of high field magnet development relevant to VLHC takes as a starting point the single aperture D20 dipole built at LBNL. D20 achieved a field of $13.5 \mathrm{~T}$ at $1.8 \mathrm{~K}$. Improving the complex and expensive design of D20 has proceeded (Fig. 3) along four interconnected paths: 1) conductor development, 2) structure improvement, 3) innovative coil geometry, and finally integrated magnet tests. The most recent success in this program is the RD3 magnet at LBNL which employs 
a $\mathrm{Nb}_{3} \mathrm{Sn}$, common coil design and new high quality superconductor to achieve $14.6 \mathrm{~T}$ at $4.5 \mathrm{~K}$. In addition, complementary programs in high-field magnet development at BNL and FNAL have emphasized high temperature superconductors and cosine coil designs respectively. At the same time the US Conductor Development Program has led to tripling of the critical current performance of $\mathrm{Nb}_{3} \mathrm{Sn}$ wire available from industry. With the newest wire of $2600 \mathrm{~A} / \mathrm{mm}^{2}$ a $15 \mathrm{~T}$ magnet is practical in the next year.

As considerable work must be done to identify the most cost-effective way of building high field magnets, and since the cost of a very large tunnel is more effectively amortized over decades of operation, the VLHC Steering Committee encouraged the development of staged deployment scenarios. Such scenarios recognize that the next tunnel may be the last one built for high energy physics. Phased scenarios inherently look at the overall plan for high energy physics over a few decades. They look for the means of incremental improvement of the collider infrastructure. In that sense they may be regarded as cost management strategies. During the past two years several phased scenarios have envisioned building multiple machines in a large tunnel (Fig. 4). One such scenario has been analyzed in considerable detail in the VLHC Design Study.

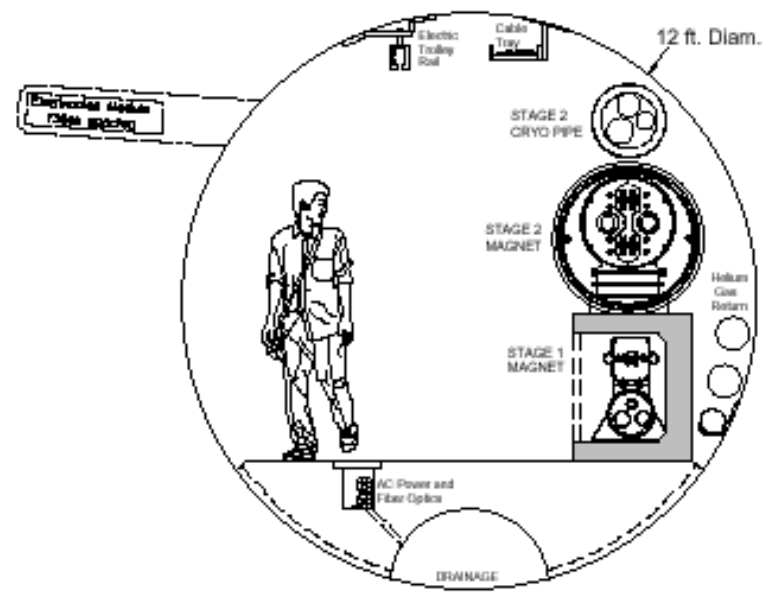

Fig.4 The 2-stage VLHC configuration

\section{VLHC DESIGN STUDY}

This year the VLHC collaboration produced an initial design of a staged VLHC in response to a request from the Fermilab director. The charge to FNAL VLHC Design Study (led by P. Limon of Fermilab) was the following:

1) Determine the characteristics of a post-LHC proton collider with initial operation at a center of mass energy $>30 \mathrm{TeV}$ and luminosity exceeding $10^{34} \mathrm{~cm}^{-}$ ${ }^{2} \mathrm{~s}^{-1}$. The design should be consistent with the option of upgrading the facility to a collider with a center of mass energy $>150 \mathrm{TeV}$ in the same tunnel.

2) Identify major challenges in accelerator technology and in the conventional construction; identify important accelerator physics issues, and unusual operational, ES\&H requirements.

3) Assuming the Fermilab complex is the injector, estimate present construction costs of the major cost drivers.

4) Identify areas of significant $R \& D$ to establish the technical basis for the facility.

The study has delivered a report (www.vlhc.org) that describes a staged approach to a VLHC in which each stage promises new and exciting particle physics. The concept is to build a big tunnel, the biggest reasonable for the site. Such a tunnel would support a collider with 20 $\mathrm{TeV}$ per beam in a $233 \mathrm{~km}$-circumference ring, based on a superferric, $2 \mathrm{~T}$ transmission line magnet design. The first stage VLHC assists in realizing the next stage by serving as a single turn injector for the higher field collider. Single turn injection reduces the aperture required in higher field magnets thereby reducing significantly the cost of the second step. A large diameter tunnel $(\sim 4 \mathrm{~m})$ was chosen to accommodate at least two collider rings. The study has addressed the practicalities of building such a large tunnel in the geology near Fermilab and assigned a tunnel cost accordingly in consultation with tunneling experts. As one sees from Table 1, each stage is a reasonable-cost step across the energy frontier.

Table 1. Parameter list for the VLHC study

\begin{tabular}{|c|c|c|}
\hline & Stage 1 & Stage 2 \\
\hline Circumference $(\mathrm{km})$ & 233 & 233 \\
\hline C-M Energy (TeV) & 40 & 175 \\
\hline Number of IRs & 2 & 2 \\
\hline Peak lum. $\left(10^{34} \mathrm{~cm}^{-2} \mathrm{~s}^{-1}\right)$ & 1 & 2 \\
\hline Lum. lifetime (hrs) 24 & 8 & \\
\hline Injection energy $(\mathrm{TeV})$ & 0.9 & 10.0 \\
\hline $\mathrm{B}_{\text {dipole }}$ at full energy $(\mathrm{T})$ & 2 & 9.8 \\
\hline Ave. arc bend rad. $(\mathrm{km})$ & 35.0 & 35.0 \\
\hline Protons/bunch $\left(10^{10}\right)$ & 2.6 & 0.8 \\
\hline Bunch Spacing (ns) & 18.8 & 18.8 \\
\hline$\beta^{*}$ at collision $(\mathrm{m})$ & 0.3 & 0.71 \\
\hline Free space in IR (m) & \pm 20 & \pm 30 \\
\hline Inelastic $\sigma(\mathrm{mb})$ & 100 & 133 \\
\hline Interactions/crossing & 21 & 58 \\
\hline $\mathrm{P}_{\text {synch }}(\mathrm{W} / \mathrm{m} /$ beam $)$ & 0.03 & 4.7 \\
\hline $\mathrm{P}_{\mathrm{ave}}$ for collider $(\mathrm{MW})$ & 20 & 100 \\
\hline Installed power (MW) & 30 & 250 \\
\hline
\end{tabular}

The study assessed the cost of the first phase of the project and did several "reality checks" of this estimate against SSC costs and TESLA cost estimates. The cost shown in Table 2 do not include contingency nor the EDIA necessary at the national laboratories to complete the project. In that sense the cost figures are directly 
comparable to the TESLA cost estimate. The conclusion is simple; "if we can afford a linear collider, we can afford the VLHC."

Naturally at this stage $R \& D$ is needed to reduce technical risk and cost, and to improve the performance of the Stage 1 collider. One sees from Table 2 that tunneling is the most expensive single part; automation is needed to reduce the labor component and make tunneling safer.

The largest technical risk factors are beam instabilities and the associated feedback systems. A combination of calculation, simulation and experiments is called for.

Magnet field quality at both injection and collision energy does not appear to be an issue, but this issue needs more study as more stringent field requires will increase dipole costs. Engineering studies of the production and handling of very long magnets may reduce cost or at least minimize the chances of costly errors. They may also find ways to reduce cost of the steel yokes and the assembly time and labor. The installation of the very long dipoles and other technical components requires a complicated, interleaved procedure that needs far more study to define acceptable options.

Vacuum systems and cryogenics turned out to be surprisingly expensive; perhaps lower cost approaches using getters can be found.

Table 2. Stage 1 costs from the VLHC Design Study

\begin{tabular}{|lcl|}
\hline $\begin{array}{l}\text { Stage-1 VLHC } \\
\text { Cost Driver }\end{array}$ & $\begin{array}{c}\text { Cost Estimate } \\
\text { (in FY2001 M\$) }\end{array}$ \\
Total Cost & 4,138 & $100 \%$ \\
Const. - Below Ground & 2,125 & $51.4 \%$ \\
Const. - Above Ground & 310 & $7.5 \%$ \\
Main Arc Magnets 792 & $19.1 \%$ & \\
Special Magnets & 112 & $2.7 \%$ \\
Refrigerators & 95 & $2.3 \%$ \\
Other Cryogenics & 22 & $0.5 \%$ \\
Installation & 232 & $5.6 \%$ \\
Vacuum System & 154 & $3.7 \%$ \\
Interaction Regions & 26 & $0.6 \%$ \\
Other Accelerator Systems & 270 & $6.5 \%$ \\
\hline
\end{tabular}

\section{CONCLUDING REMARKS}

Several exhortations serve to conclude this summary of the status of VLHC research. Include high quality system engineering at the outset and engage industry early in value engineering. Think through all the consequences of technical choices. Conduct thorough fault mode analysis. For example, analyze where stored energy is more risky - in the magnets or in the beam. Be imaginative and consider where designer materials might reduce cost spent dramatically. Design in production reliability; a few dollars in advance will lead to reduced fabrication costs. Don't invent every piece of technology anew; look for analog industrial experience in commercial markets.

A final word: the public is part of the project. Taxpayers pay the cost; they must share the excitement. We can connect to the public's cosmic fascination with our search for hidden universes (extra dimensions).

The SSC experience should teach us not to take the broader physics community for granted, either. Our community must articulate the intellectual excitement to those in other disciplines. Perhaps they "get a piece" (such as the FEL in the TESLA proposal). Inclusion of other scientific communities from the beginning minimizes surprises in either direction.

In other words, "sticker-shock" had better not precede excitement. High energy physicists have a notorious habit of intellectual pride; my advice is to leave it back at the lab; there are no "C students," especially not on Capitol Hill.

\section{ACKNOWLEDGEMENTS}

I am indebted to my fellow members of the VLHC Steering Committee (Peter Limon, Ernest Malamud, Steve Peggs, Mike Harrison and Jim Siegrist) and to Bill Foster and Alan Jackson for many hours of thought provoking discussions. The output of the several VLHC workshops posted on the collaboration web page have been a source of valuable insight. The VLHC Design Study Report has been a crucial resource; I urge everyone to read it. Many of the concepts for the development of the high field magnet program have been formulated and discussed over the past few years at a series of Eloisatron workshops held at the Ettore Majorana Center for Scientific Culture in Erice, Sicily. I am grateful to Prof. Antonino Zichichi for his support of these meetings; he has inspired my enthusiasm for the $200 \mathrm{TeV}$ frontier. This work was supported by the Office of High Energy Physics of the U.S. Department of Energy under Contract No. DEAC03-76SF00098. 